Research Paper

\title{
A Multicenter Retrospective Comparison of Sequential versus Sandwich Chemoradiotherapy for Stage IE-IIE Extranodal Natural Killer/T-Cell Lymphoma, Nasal Type
}

\author{
Jin $\mathrm{Li}^{1}$, Yajun Li ${ }^{1,2}$, Meizuo Zhong 3 , Wei Liu ${ }^{3}$, Xianling Liu ${ }^{4}$, Jiwei $\mathrm{Li}^{1}$, Kunlun Li1, Pingyong Yi1,2, \\ 1. Department of Lymphoma and Hematology, The Affiliated Cancer Hospital of Xiangya School of Medicine, Central South University, Changsha, Hunan, \\ China, 410013. \\ 2. Department of Lymphoma and Hematology, Hunan Cancer Hospital, Changsha, Hunan, China, 410013. \\ 3. Department of Oncology, Xiangya Hospital, Central South University, Changsha, Hunan, China, 410008. \\ 4. Department of Oncology, The Second Xiangya Hospital, Central South University, Changsha, Hunan, China, 410011. \\ $\triangle$ Corresponding author: Prof. Pingyong Yi, Department of Lymphoma and Hematology, Hunan Cancer Hospital, 283 Tongzipo Road, Yuelu District, \\ Changsha 410013, Hunan, China; Tel: +86-137 8616 1716; Email: yipingyong1@163.com \\ (c) Ivyspring International Publisher. This is an open access article distributed under the terms of the Creative Commons Attribution (CC BY-NC) license \\ (https://creativecommons.org/licenses/by-nc/4.0/). See http://ivyspring.com/terms for full terms and conditions.
}

Received: 2017.12.12; Accepted: 2018.03.03; Published: 2018.04.12

\begin{abstract}
Background: Chemotherapy and radiotherapy are critical for treating early-stage extranodal natural killer/T-cell lymphoma, nasal type (ENKTL); however, the optimal therapy sequence remains unclear. Therefore, we performed this study to compare the efficacy of L-asparaginase/pegaspargase-based sequential versus sandwich chemoradiotherapy for patients newly diagnosed with stage IE-IIE ENKTL.

Methods: Patients were categorized into sequential $(N=111)$ and sandwich $(N=104)$ groups. Chemotherapy regimens included GELOX, SMILE, and VLP. The median radiotherapy dose was 55.0 Gy (range, 40.0-63.0 Gy). Adverse events, treatment responses, and survival outcomes were analyzed.

Results: Patients' clinical characteristics were largely comparable between the 2 groups; however, the sandwich group comprised a larger number of Ann Arbor stage IIE patients. Local invasion was the most significant predictor of overall survival (OS); local invasion and Ann Arbor stage were significant predictors of progression-free survival (PFS). There were no significant differences in the complete response rate $(85.6 \%$ vs. $89.4 \%, p=0.396)$, 3 -year OS $(77.5 \%$ vs. $80.8 \%, p=0.636)$, or 3 -year PFS rates $(74.8 \%$ vs. $76.9 \%, p=0.806)$ in the sequential vs. sandwich groups, respectively. The incidence of grade $3 / 4$ hematological toxicities was higher in the sandwich group than in the sequential group $(27.9 \%$ vs. $15.3 \%$, respectively, $p=0.025)$. The response rates and survival outcomes in stage IE and IIE patients did not differ between sequential and sandwich groups.

Conclusions: In the era of L-asparaginase/pegaspargase, both sequential and sandwich chemoradiotherapy are safe and similarly effective in patients with newly diagnosed stage IE-IIE ENKTL.
\end{abstract}

Key words: Extranodal natural killer/T-cell lymphoma, nasal type; sequential chemoradiotherapy; sandwich chemoradiotherapy

\section{Introduction}

Extranodal natural killer/T-cell lymphoma, nasal type (ENKTL) is a form of non-Hodgkin's lymphoma with a low incidence and a geographical predilection to Asia and Latin America [1]. ENKTL commonly involves the nasal and paranasal area as well as the upper respiratory and digestive tracts. Approximately two-thirds of cases are stage IE-IIE [2, $3]$. The disease has a poor prognosis and an aggressive 
clinical course; the 5-year OS rate of patients with early-stage ENKTL is reportedly 38-73\% [4-6].

For patients with early-stage ENKTL, radiotherapy alone leads to CR rates of $50-100 \%$ [4, 7-9], but also carries a high risk of systemic failure in $25-40 \%$ of patients with limited-stage disease [7]. This suggests that radiotherapy should be combined with chemotherapy to reduce the incidence of distant recurrence.

ENKTL is insensitive to traditional anthracycline-based regimens, as more than $60 \%$ of patients become resistant to such therapies [10-13]. The high expression of multi-drug resistance (MDR) genes in ENKTL may lead to poor responses to the conventional CHOP regimen [14]. The addition of Lasparaginase/pegaspargase, which is not influenced by MDR genes, has improved the survival rates of patients with ENKTL $[15,16]$. L-asparaginase-based regimens have emerged as potential standard treatments for this condition [17]; some of these regimens have achieved CR rates of $40-60 \%$ [18-20].

Current effective therapeutic strategies for stage IE-IIE ENKTL include concurrent chemoradiotherapy (CCRT) followed by chemotherapy, sequential chemoradiotherapy, and sandwich chemoradiotherapy [21]. Several phase II single-arm clinical trials have demonstrated the promising effects of these treatment modalities. The CR rates following CCRT, sequential chemoradiotherapy, and sandwich chemoradiotherapy are $80-87 \%, 91-100 \%$, and $74-81 \%$, respectively; the corresponding 5-year OS rates are $72-86 \%, 86-89 \%$, and $64-85 \%$, respectively [22-30].

Although chemotherapy and radiotherapy are essential for treating early-stage ENKTL, no prospective studies to compare the efficacy of L-asparaginase-based sequential chemoradiotherapy with sandwich chemoradiotherapy have been performed, and the optimal sequence of chemotherapy and radiotherapy has yet to be determined. Therefore, we conducted this multicenter retrospective analysis to compare the objective response rates and survival outcomes between these 2 treatment modalities in patients with ENKTL.

\section{Materials and Methods}

\section{Patients}

We retrospectively reviewed the clinical data of 215 treatment-naïve patients with stage IE-IIE ENKTL who attended the Hunan Cancer Hospital, Xiangya Hospital of Central South University or Second Xiangya Hospital of Central South University between 2010 and 2017. ENKTL was diagnosed according to the current World Health Organization classification [31, 32]. All patients underwent routine blood examinations; blood biochemistry; bone marrow smear and biopsy; CT or MRI of the involved areas; a CT scan of the neck, chest, abdomen, and pelvis; and/or whole-body PET/CT prior to treatment.

Patient characteristics including age, gender, ECOG performance status, Ann Arbor stage, lactate dehydrogenase (LDH) levels, plasma EBV DNA level, B symptoms, PINK and PINK-E scores, local invasion, chemotherapy regimens, and radiotherapy data were collected. Local tumor invasion was defined as occurring when tumors spanned neighboring structures (such as the nasal skin, paranasal sinus, orbit, and hard or soft palate) by contiguous spreading. Stage and risk stratification were determined according to the Ann Arbor stage [33, 34] and PINK/PINK-E scores [35], respectively. Early-stage, limited-stage, and localized ENKTL referred to Ann Arbor stages IE or IIE.

The inclusion criteria were as follows: (1) pathological diagnosis of ENKTL, (2) newly diagnosed patients, (3) Ann Arbor stage IE-IIE, (4) underwent $\geq 1$ cycle of L-asparaginase-based chemotherapy, and (5) underwent radiotherapy. Patients were excluded if they had coexisting malignant tumors, severe organ dysfunction, or any concomitant medical condition that may have caused poor compliance with the research protocol.

All participants provided informed written consent. The study protocol was approved by the Institutional Review Board of the National Cancer Institute and the Ethics Committee of each of the above hospitals. This research was conducted in accordance with the 2008 Declaration of Helsinki.

\section{Treatment and response criteria}

All patients were treated with combined L-asparaginase/pegaspargase-based chemotherapy and radiotherapy. The treatment modalities were categorized into 2 groups based on the timing of radiotherapy in relation to the chemotherapy: the sequential group (induction chemotherapy followed by radiotherapy, or radiotherapy followed by consolidation chemotherapy) and the sandwich group (induction chemotherapy followed by radiotherapy, and then by consolidation chemotherapy). The chemotherapy regimens consisted of GELOX, SMILE, and VLP; the regimens and dosages are listed in Table 1 . The response criteria were evaluated according to the Lugano Response Criteria for non-Hodgkin's lymphoma [34]. Tumor responses were assessed through physical examinations, nasopharyngoscopy, CT, MRI, and/or PET/CT every 2 cycles during chemotherapy and 1 month after radiotherapy. 
Table 1. Chemotherapy regimens

\begin{tabular}{lll}
\hline Regimen & Drugs & Dosage \\
\hline GELOX & gemcitabine & $800 \mathrm{mg} / \mathrm{m}^{2}, \mathrm{~d} 1$ and $\mathrm{d} 8$ \\
& oxaliplatin & $85 \mathrm{mg} / \mathrm{m}^{2}, \mathrm{~d} 1$ \\
& L-asparaginase/pegaspargase & $5000 \mathrm{U} / \mathrm{m}^{2}, \mathrm{~d} 1-7 / 2500 \mathrm{IU} / \mathrm{m}^{2}, \mathrm{~d} 1$ \\
SMILE & dexamethasone & $15 \mathrm{mg}, \mathrm{d} 1-7$ \\
& methotrexate & $60 \mathrm{mg} / \mathrm{m}^{2}, \mathrm{~d} 1$ \\
& ifosfamide & $1.5 \mathrm{~g} / \mathrm{m}^{2}, \mathrm{~d} 2-4$ \\
& L-asparaginase/pegaspargase & $5000 \mathrm{U} / \mathrm{m}^{2}, \mathrm{~d} 1-7 / 2500 \mathrm{IU} / \mathrm{m}^{2}, \mathrm{~d} 1$ \\
& etoposide & $100 \mathrm{mg} / \mathrm{m}^{2}, \mathrm{~d} 2-4$ \\
VLP & vincristine & $2 \mathrm{mg}, \mathrm{d} 1$ \\
& L-asparaginase/pegaspargase & $5000 \mathrm{U} / \mathrm{m}^{2}, \mathrm{~d} 1-7 / 2500 \mathrm{IU} / \mathrm{m}^{2}, \mathrm{~d} 1$ \\
& prednisone & $100 \mathrm{mg} / \mathrm{d}, \mathrm{d} 1-5$ \\
\hline
\end{tabular}

Treatment-related toxicity was evaluated according to the National Cancer Institute's Common Toxicity Criteria (version 3) [36].

\section{Statistical analyses}

OS was calculated from the date of diagnosis to that of death or the last follow-up date. PFS was defined as the interval between the date of diagnosis and the date of disease progression, relapse, or death by any cause. Differences in patient clinical characteristics, response rates, recurrence rates, and mortality rates between the sequential and sandwich groups were evaluated using the Chi-square or Kruskal-Wallis test. Survival analysis was performed using the Kaplan-Meier method with the log-rank test. Prognostic factors for OS and PFS were analyzed using Cox regression analysis. All statistical analyses were used SPSS 24.0 software (IBM Corp., Armonk, NY, USA). A $p$-value $<0.05$ was considered statistically significant.

\section{Results}

\section{Patient characteristics}

Between 2010 and 2017, a total of 215 patients with stage IE-IIE ENKTL were enrolled in this retrospective study. These patients were predominantly male (the male: female ratio was $2.6: 1$ ). The median patient age was 44 (range, 14-77) years. In terms of treatment, 111 patients were in the sequential group and 104 in the sandwich group. Seventy-eight patients in the sequential group received chemotherapy followed by radiotherapy $(\mathrm{CT}+\mathrm{RT})$ while 33 received radiotherapy followed by chemotherapy $(\mathrm{RT}+\mathrm{CT})$.

The baseline clinical characteristics, including age $(p=0.068)$, gender $(p=0.869)$, ECOG performance status $(p=0.523)$, LDH levels $(p=0.735)$, B symptoms $(p=0.805)$, PINK scores $(p=0.190)$, PINK-E scores $(p=$ $0.449)$, and local invasion $(p=0.317)$, were largely comparable between the 2 groups. However, significantly more patients with Ann Arbor stage IIE disease were included in the sandwich group (32.7\%) than in the sequential group $(19.8 \% ; p=0.032)$ (Table 2).
Table 2. Patient characteristics

\begin{tabular}{lllll}
\hline Characteristic & \multicolumn{1}{l}{ Sequential Group } & Sandwich Group & $p$ & All n (\%) \\
\hline Gender & & & 0.869 & \\
Male & $80(72.1)$ & $76(73.1)$ & & $156(72.6)$ \\
Female & $31(27.9)$ & $28(26.9)$ & & $59(27.4)$ \\
Age & & & 0.068 & \\
$>60$ & $13(11.7)$ & $5(4.8)$ & & $18(8.4)$ \\
$\leq 60$ & $98(88.3)$ & $99(95.2)$ & & $197(91.6)$ \\
ECOG & & & 0.523 & \\
$0-1$ & $110(99.1)$ & $102(98.1)$ & & $212(98.6)$ \\
$2-4$ & $1(0.9)$ & $2(1.9)$ & & $3(1.4)$ \\
Ann Arbor stage & & & 0.032 & \\
IE & $89(80.2)$ & $70(67.3)$ & & $159(74.0)$ \\
IIE & $22(19.8)$ & $34(32.7)$ & & $56(26.0)$ \\
LDH & & & 0.735 & \\
Elevated & $30(27.0)$ & $26(25.0)$ & & $56(26.0)$ \\
Normal & $81(73.0)$ & $78(75.0)$ & & $159(74.0)$ \\
B symptoms & & & 0.805 & \\
Present & $68(61.3)$ & $62(59.6)$ & & $130(60.5)$ \\
Absent & $43(38.7)$ & $42(40.4)$ & & $85(39.5)$ \\
PINK & & & 0.190 & \\
Low-risk & $94(84.7)$ & $96(92.3)$ & & $190(88.4)$ \\
Intermediate-risk & $16(14.4)$ & $7(6.7)$ & & $23(10.7)$ \\
High-risk & $1(0.9)$ & $1(1.0)$ & & $2(0.9)$ \\
PINK-E & & & 0.449 & \\
Low-risk & $45(91.8)$ & $42(93.4)$ & & $87(92.5)$ \\
Intermediate-risk & $4(8.2)$ & $2(4.4)$ & & $6(6.4)$ \\
High-risk & $0(0.0)$ & $1(2.2)$ & & $1(1.1)$ \\
Local invasion & & & 0.317 & \\
$\begin{array}{l}\text { Present } \\
\text { Absent }\end{array}$ & $62(55.9)$ & $51(49.0)$ & & $113(52.6)$ \\
Chemotherapy regimen & $84(74.1)$ & $53(51.0)$ & & $102(47.4)$ \\
GELOX & $84.7)$ & $82(78.8)$ & & $166(77.2)$ \\
SMILE & $11(9.9)$ & $11(10.6)$ & & $22(10.2)$ \\
VLP & $16(14.4)$ & $11(10.6)$ & & $27(12.6)$ \\
\hline & & & &
\end{tabular}

Primary tumor sites included the nasal cavities $(N=159$; sequential group $N=79$ and sandwich group $N=80)$, upper aerodigestive tract $(N=52$; sequential group $N=29$ and sandwich group $N=23$ ), and non-upper aerodigestive tract $(N=4$; sequential group $N=3$ and sandwich group $N=1$ ). Plasma EBV-DNA levels were tested in 94 patients and were detectable in 45 (22 in the sequential group and 23 in the sandwich group).

\section{Adverse events}

The majority of patients tolerated any treatment-related adverse events that occurred, and there were no treatment-related deaths. Neutropenia and radiation-induced mucositis were the most common hematological and non-hematological adverse events, respectively. The incidences of grade $1 / 2$ hematological toxicities and grade $1 / 2$ and $3 / 4$ non-hematological toxicities did not differ significantly between the 2 groups. However, the incidence of grade $3 / 4$ hematological toxicities was significantly higher in the sandwich group than in the sequential group $(27.9 \%$ vs. $15.3 \%, p=0.025)$ (Table 3$)$.

\section{Treatment responses}

The median radiotherapy dose for both the sequential and sandwich groups was 55.0 Gy (ranges, 40.0-63.0 and 50.0-63.0 Gy, respectively). All patients 
received a median of 4 (range, 1-8) cycles of chemotherapy. In the sequential group, 84 patients (75.7\%) were treated with the GELOX regimen (1-7 cycles), 11 (9.9\%) with the SMILE regimen (2-8 cycles), and 16 (14.4\%) with the VLP regimen (1-6 cycles). In the sandwich group, 82 patients $(78.8 \%)$ were treated with the GELOX regimen ( $2-6$ cycles), 11 (10.6\%) with the SMILE regimen (3-7 cycles), and 11 $(10.6 \%)$ with the VLP regimen (2-6 cycles). The proportions of patients receiving each regimen were balanced between the 2 groups.

Table 3. Adverse events

\begin{tabular}{lllllll}
\hline \multicolumn{1}{c}{ Grade 1-2 } & \multicolumn{5}{c}{ Grade 3-4 } \\
\hline Adverse events & $\begin{array}{l}\text { Sequential } \\
\text { group }\end{array}$ & $\begin{array}{l}\text { Sandwich } \\
\text { group }\end{array}$ & $p$ & $\begin{array}{l}\text { Sequential } \\
\text { group }\end{array}$ & $\begin{array}{l}\text { Sandwich } \\
\text { group }\end{array}$ & $p$ \\
\hline Hematological & $73(65.8 \%)$ & $68(65.4 \%)$ & 0.953 & $17(15.3 \%)$ & $29(27.9 \%)$ & 0.025 \\
Neutropenia & $41(36.9 \%)$ & $47(45.2 \%)$ & 0.219 & $17(15.3 \%)$ & $27(26.0 \%)$ & 0.053 \\
Anemia & $50(45.0 \%)$ & $47(45.2 \%)$ & 0.983 & $2(1.8 \%)$ & $2(1.9 \%)$ & 0.948 \\
Thrombocytopenia & $17(15.3 \%)$ & $13(12.5 \%)$ & 0.552 & $6(5.4 \%)$ & $5(4.8 \%)$ & 0.842 \\
$\begin{array}{l}\text { Non-hematologica } \\
\text { l }\end{array}$ & $60(54.1 \%)$ & $57(54.8 \%)$ & 0.912 & $21(18.9 \%)$ & $18(17.3 \%)$ & 0.759 \\
$\begin{array}{l}\text { Nausea/Vomiting } \\
\text { Transaminase }\end{array}$ & $35(31.5 \%)$ & $41(39.4 \%)$ & 0.226 & $6(5.4 \%)$ & $8(7.7 \%)$ & 0.497 \\
elevation & $47(42.3 \%)$ & $42(40.4 \%)$ & 0.771 & $4(3.6 \%)$ & $2(1.9 \%)$ & 0.455 \\
$\begin{array}{l}\text { Radio-mucositis } \\
\text { Radio-dermatitis }\end{array}$ & $37(33.3 \%)$ & $35(33.7 \%)$ & 0.960 & $12(10.8 \%)$ & $12(11.5 \%)$ & 0.866 \\
& $16(14.4 \%)$ & $22(21.2 \%)$ & 0.195 & $3(2.7 \%)$ & $2(1.9 \%)$ & 0.705
\end{tabular}

Across the entire cohort, CR, PR, and PD were observed in 188 (87.4\%), 14 (6.5\%), and $13(6.1 \%)$ patients, respectively, after the completion of treatment. After the initial chemotherapy, CR, PR, SD, and PD were observed in $53(47.7 \%), 56$ (50.5\%), 1 $(0.9 \%)$, and $1(0.9 \%)$ patient(s), respectively, in the sequential group. Following sequential chemoradiotherapy, CR, PR, and PD were observed in 95 (85.6\%), $7(6.3 \%)$, and $9(8.1 \%)$ patients, respectively. Forty-three patients showed a change from PR to CR, 1 from SD to CR, and 1 from PD to CR. Moreover, 3 patients showed a change from CR to PD and 6 from $\mathrm{PR}$ to $\mathrm{PD}$. The remaining 57 patients maintained the initial response. In the sandwich group, CR and PR

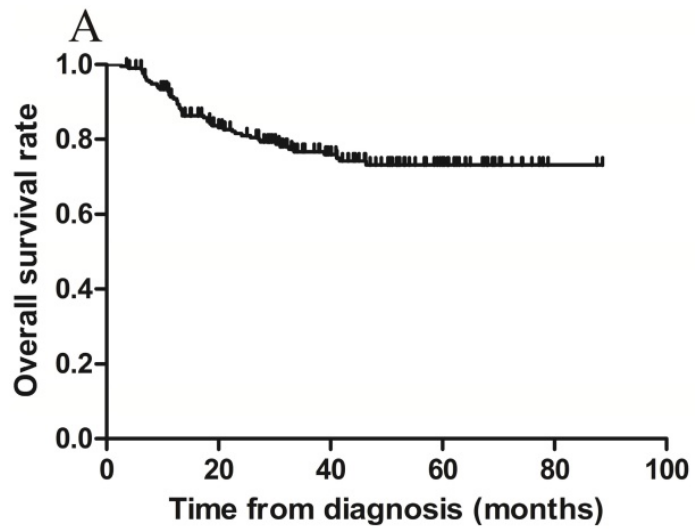

were observed in $39(37.5 \%)$ and $65(62.5 \%)$ patients, respectively, after the initial chemotherapy. Following sandwich chemoradiotherapy, CR, PR, and PD were observed in $93(89.5 \%), 7$ (6.7\%), and 4 (3.8\%) patients, respectively. Thirty-eight patients showed a change from PR to CR and 1 from PR to SD. The remaining 65 patients maintained the initial response. CR rates did not differ significantly between the 2 groups (85.6\% vs. $89.4 \%$ for the sequential and sandwich groups, respectively; $p=0.396$ ).

\section{Treatment failure}

Thirty-seven patients (sequential group $N=17$ and sandwich group $N=20$ ) experienced recurrence during the follow-up period. Nineteen patients developed regional recurrence (sequential group $N=$ 8 and sandwich group $N=11$ ) and 18 developed distant recurrence (sequential group $N=9$ and sandwich group $N=9$ ). There was no significant difference in recurrence rates between the sequential and sandwich groups (15.3\% vs. $19.2 \%$, respectively, p $=0.447$ ).

\section{Survival and prognosis}

The median follow-up time for all patients was 35.1 (range, 2.6-88.6) months as of October 2017. The median follow-up time for the sequential and sandwich groups were 33.1 (range, 2.6-78.9) and 38.4 (range, 3.6-88.6) months, respectively. The mortality rates were $22.5 \%(N=25$ patients) and $23.1 \%(N=24$ patients), respectively, and were comparable between the 2 treatment modalities $(p=0.923)$.

The 3-year OS and PFS rates for all patients combined were $79.1 \%$ and $75.8 \%$, respectively (Figure 1 A-B). The 3-year OS rates for the sequential and sandwich groups were $77.5 \%$ and $80.8 \%$, respectively; the 3-year PFS rates were $74.8 \%$ and $76.9 \%$, respectively (Figure 2A-B). No significant differences in the 3-year OS or PFS rates were observed between the 2 groups ( $p=0.636$ and $p=0.806$, respectively).

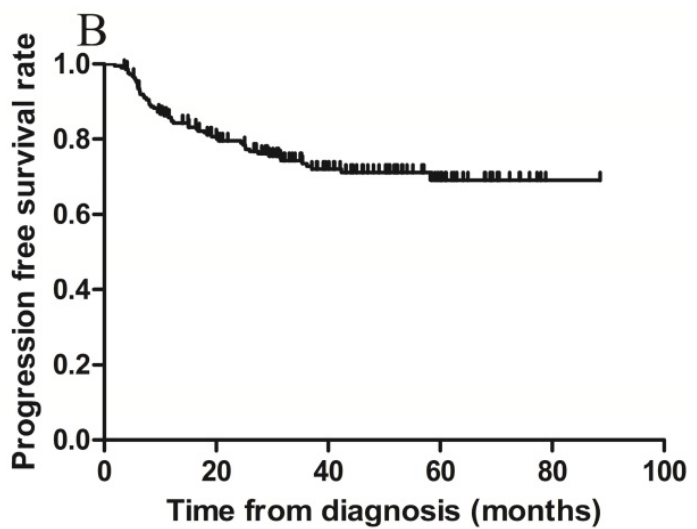

Figure 1. Kaplan-Meier survival curves for all patients. A) Overall survival; the 3-year rate was 79.1\%. B) Progression-free survival; the 3-year rate was 75.8\%. 

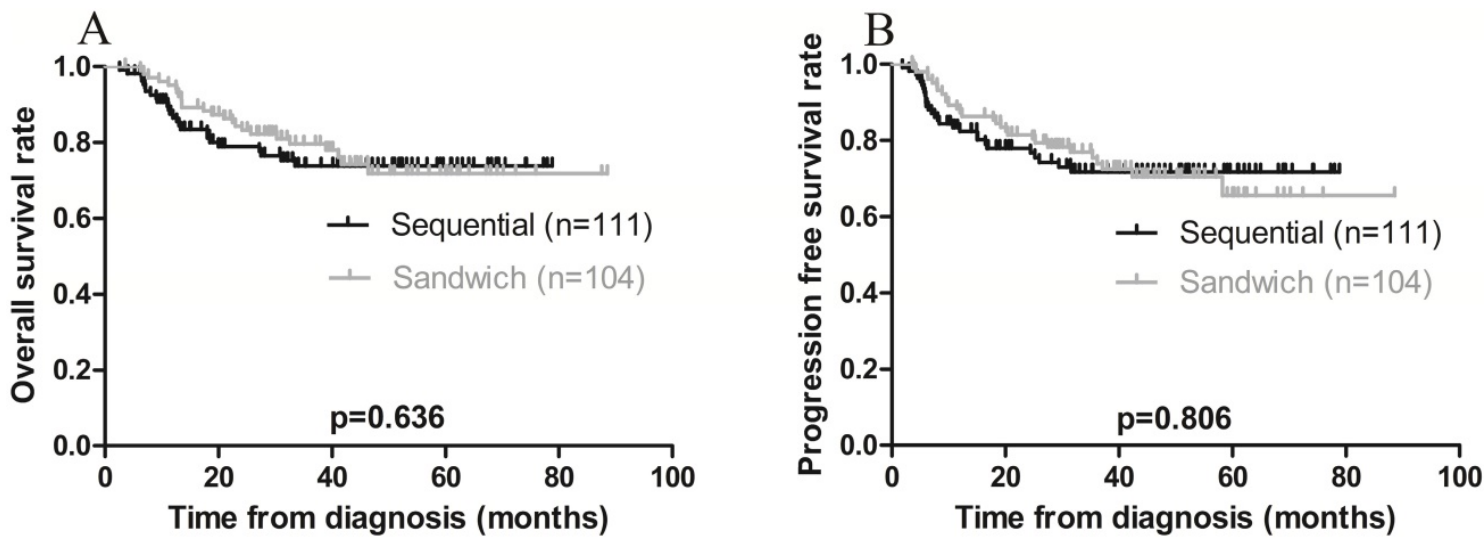

Figure 2. Kaplan-Meier survival curves plotted according to treatment modality. A) Overall survival; the 3 -year rates in the sequential and sandwich groups were $77.5 \%$ and $80.8 \%$, respectively $(p=0.636)$. B) Progression-free survival; the 3 -year rates in the sequential and sandwich groups were $74.8 \%$ and $76.9 \%$, respectively $(p=0.806)$.
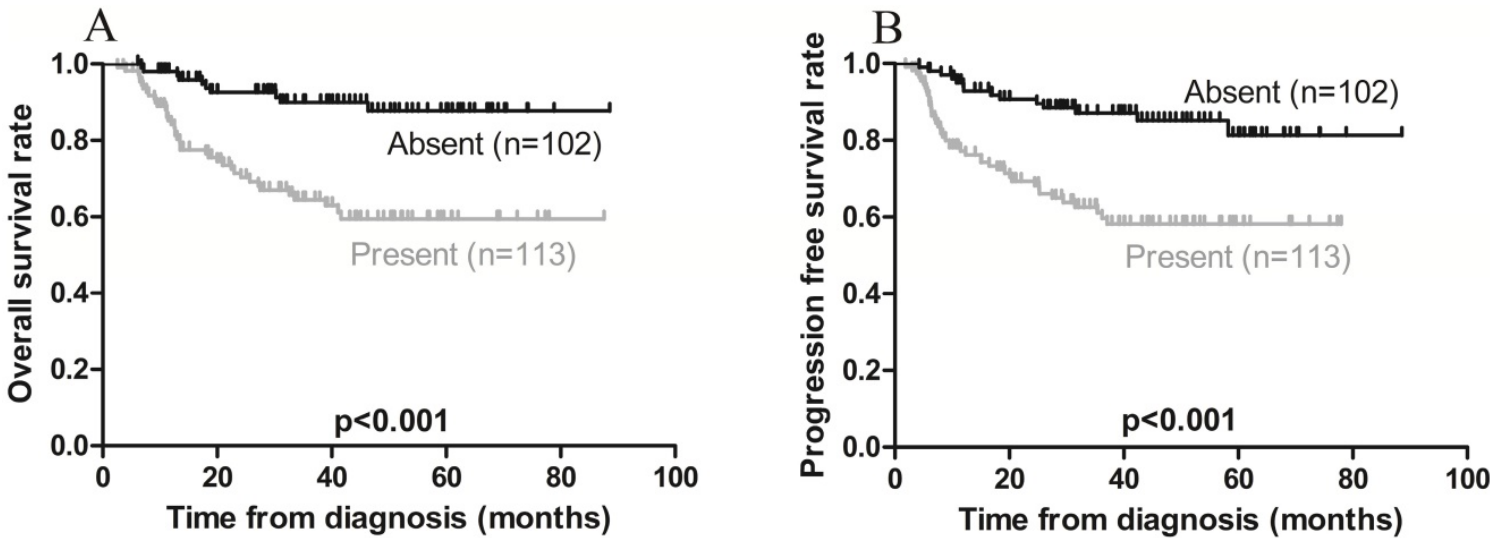

Figure 3. Kaplan-Meier survival curves in patients with (present) and without (absent) local invasion. A) Overall survival; the 3-year rates in the present and absent groups were $68.1 \%$ and $91.2 \%$, respectively $(p<0.001)$. B) Progression-free survival; the 3 -year rates in the present and absent groups were $64.6 \%$ and 88.2 , respectively $(p<0.001)$.
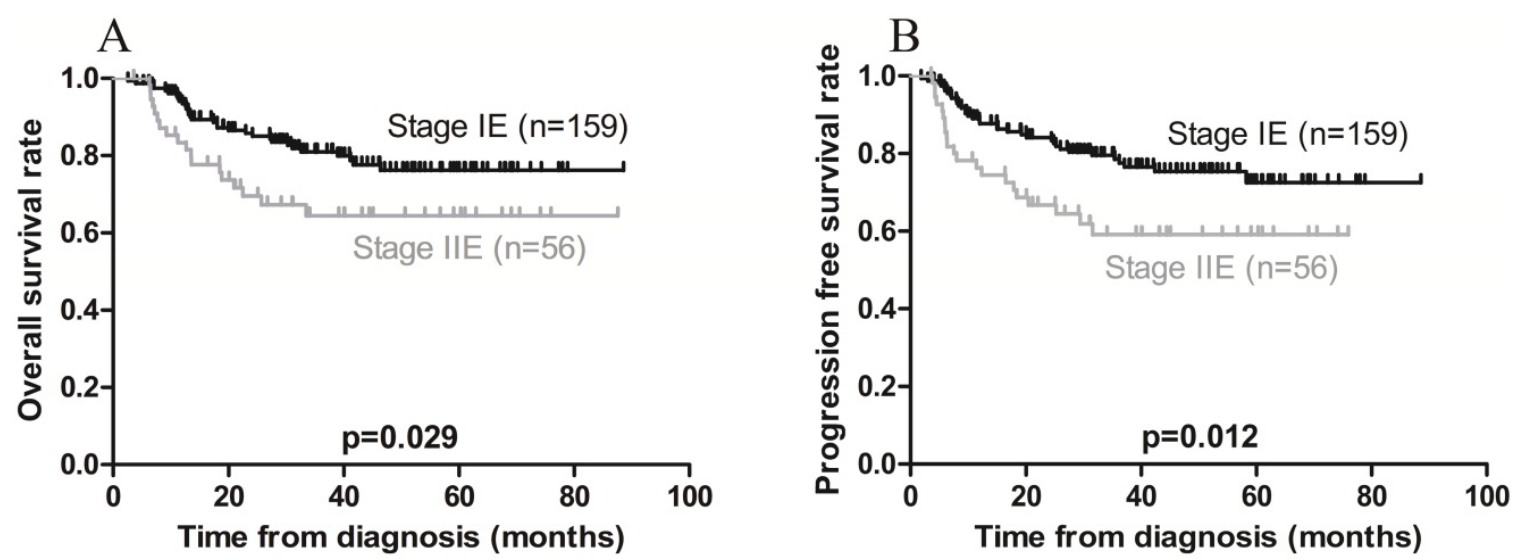

Figure 4. Kaplan-Meier survival curves in patients with Ann Arbor stage IE and IIE extranodal natural killer/T-cell lymphoma, nasal type. A) Overall survival; the 3-year rates in patients with stage IE and stage IIE disease were $83.0 \%$ and $67.9 \%$, respectively $(p=0.029)$. B) Progression-free survival; the 3 -year rates in patients with stage IE and stage IIE disease were $80.5 \%$ and $62.5 \%$, respectively $(p=0.012)$.

On univariate analysis, local invasion adversely affected OS, while Ann Arbor stage IE was associated with a favorable OS (Figures $3 \mathrm{~A}$ and 4A). On multivariate analysis, local invasion (HR: 0.246, 95\% CI: $0.122-0.494 ; p<0.001)$ was the most significant predictor of shorter OS (Table 4).

Furthermore, univariate analysis revealed that local invasion was associated with poorer PFS, while
Ann Arbor stage IE was associated with a favorable PFS (Figures 3B and 4B). On multivariate analysis, local invasion (HR: 0.317, 95\% CI: 0.172-0.583; $p<$ 0.001 ) and Ann Arbor stage IIE (HR: 1.732, 95\% CI: 1.004-2.987; $p=0.048$ ) were the most significant predictors of shorter PFS (Table 4). 
Table 4. Univariate and multivariate analyses for factors affecting survival outcomes

\begin{tabular}{|c|c|c|c|c|c|c|c|c|}
\hline \multirow[t]{3}{*}{ Clinical factor } & \multicolumn{4}{|c|}{ Overall survival } & \multicolumn{4}{|c|}{ Progression-free survival } \\
\hline & \multicolumn{2}{|c|}{ Univariate } & \multicolumn{2}{|c|}{ Multivariate } & \multicolumn{2}{|c|}{ Univariate } & \multicolumn{2}{|c|}{ Multivariate } \\
\hline & $p$ & $\mathrm{HR}(95 \% \mathrm{CI})$ & $p$ & HR $(95 \% \mathrm{CI})$ & $p$ & HR $(95 \% \mathrm{CI})$ & $p$ & HR $(95 \% \mathrm{CI})$ \\
\hline Female & 0.061 & $0.485(0.227-1.035)$ & & & 0.081 & $0.544(0.274-1.077)$ & & \\
\hline Age $\leq 60$ & 0.088 & $0.498(0.224-1.109)$ & & & 0.055 & $0.481(0.227-1.017)$ & & \\
\hline ECOG 0-1 & 0.476 & $0.487(0.067-3.531)$ & & & 0.546 & $0.544(0.075-3.934)$ & & \\
\hline $\begin{array}{l}\text { Ann Arbor } \\
\text { stage IIE }\end{array}$ & 0.029 & $1.892(1.058-3.385)$ & & & 0.012 & $1.982(1.153-3.407)$ & 0.048 & $1.732(1.004-2.987)$ \\
\hline Normal LDH & 0.811 & $0.927(0.499-1.723)$ & & & 0.777 & $0.920(0.515-1.643)$ & & \\
\hline $\begin{array}{l}\text { Absent } \\
\text { B symptoms }\end{array}$ & 0.876 & $0.955(0.538-1.697)$ & & & 0.975 & $0.991(0.580-1.695)$ & & \\
\hline PINK Intermediate-risk & 0.165 & $1.711(0.802-3.650)$ & & & 0.140 & $1.713(0.839-3.498)$ & & \\
\hline PINK-E Intermediate-risk & 0.249 & $2.425(0.537-10.951)$ & & & 0.285 & $2.265(0.507-10.122)$ & & \\
\hline Absent local invasion & $<0.001$ & $0.233(0.116-0.467)$ & $<0.001$ & $0.246(0.122-0.494)$ & $<0.001$ & $0.299(0.163-0.548)$ & $<0.001$ & $0.317(0.172-0.583)$ \\
\hline Sandwich chemoradiotherapy & 0.636 & $1.070(0.808-1.417)$ & & & 0.806 & $1.033(0.795-1.343)$ & & \\
\hline
\end{tabular}

\section{Subgroup analysis}

Among the clinical characteristics examined, only Ann Arbor stage was not balanced between the 2 groups, which is important because Ann Arbor stage notably affected the patients' prognoses. Therefore, we subsequently performed a subgroup analysis of the Ann Arbor stage. Regardless of stage IE or IIE, clinical characteristics between the sequential and sandwich groups were balanced (Table S1 and S2). In stage IE patients, the rates of CR, recurrence, 3-year OS, and 3-year PFS in the sequential and sandwich groups were $88.8 \%$ vs. $91.4 \%(p=0.579), 13.5 \%$ vs. $17.1 \%(\mathrm{p}=0.522), 80.9 \%$ vs. $85.7 \%(\mathrm{p}=0.637)$, and $79.8 \%$ vs. $81.4 \%(\mathrm{p}=0.991)$, respectively. In stage IIE patients, these rates were $72.7 \%$ vs. $85.3 \%(p=0.248)$, $22.7 \%$ vs. $23.5 \%(\mathrm{p}=0.945), 63.6 \%$ vs. $70.6 \%(\mathrm{p}=$ 0.497 ), and $54.5 \%$ vs. $67.6 \%$ ( $\mathrm{p}=0.356$ ), respectively. Overall, there were no significant differences in objective responses rates and survival outcomes between the 2 groups regardless of stage IE or IIE disease.

Additional analysis was performed to compare the efficacy of RT+CT to that of CT+RT. The clinical features of these 2 groups were balanced (Table S3). In the $\mathrm{RT}+\mathrm{CT}$ group, the initial radiotherapy achieved a CR rate of $66.7 \%$ ( $N=22$ patients); in contrast, the CR rate after the initial chemotherapy in the CT+RT group was only $35.9 \%(N=28)$. However, the $C R$ rates after completion of chemoradiotherapy were not significantly different between the 2 groups $(90.9 \%$ vs. $83.3 \%$ for the $\mathrm{RT}+\mathrm{CT}$ and $\mathrm{CT}+\mathrm{RT}$ groups, respectively; $p=0.299$ ). The rates of recurrence, 3-year OS, and 3-year PFS also did not differ between the RT+CT and CT+RT groups: $15.2 \%$ vs. $15.4 \%(p=0.975), 81.8 \%$ vs. $75.6 \%(p=0.291)$, and $78.8 \%$ vs. $73.1 \%(p=0.374)$, respectively. Taken together, the therapeutic responses and survival outcomes were comparable between the 2 groups.

\section{Discussion}

Our study revealed local invasion to be the most significant predictor of poor OS, while both local invasion and Ann Arbor stage IIE are the most significant predictors of poor PFS. For all patients combined, the CR rate as well as the 3-year OS and PFS rates did not significantly differ between the sequential and sandwich groups. Moreover, the response rates and survival outcomes were not significantly different for patients with stage IE and IIE disease between the chemoradiotherapy modalities. Our findings suggest that, as long as radiotherapy and chemotherapy are used in combination, the sequence of treatment may not influence the therapeutic efficacy and survival outcomes in patients with localized ENKTL.

Currently, there are no standard treatment strategies for early-stage ENKTL. CHOP and CHOP-like regimens have proved to be ineffective for ENKTL patients with high recurrence rates and short survival times [10, 11, 13]. Furthermore, several studies [37-39] have demonstrated that L-asparaginase/pegaspargase-based regimens are superior to traditional $\mathrm{CHOP}$ or $\mathrm{CHOP}$-like regimens in terms of response rates and survival outcomes. The recommended treatment for limited-stage ENKTL is radiotherapy combined with L-asparaginase/ pegaspargase-based chemotherapy. A prospective open-label controlled trial recruited 427 ENKTL patients, of whom 202 received chemoradiotherapy (using cyclophosphamide + methotrexate + etoposide + dexamethasone), 109 received radiotherapy alone, and 116 received the aforementioned chemotherapy regimen alone. Chemoradiotherapy significantly enhanced the CR rate compared to radiotherapy or chemotherapy alone $(91 \%$ vs. $69 \%$ vs. $59 \%$, respectively; $p<0.01)$, as it did the 5 -year OS rate $(86 \%$ vs. $64 \%$ vs. $45 \%$, respectively; $p<0.001$ ) [27]. A retrospective cohort study of 33 patients treated with chemoradiotherapy (using L-asparaginase + 
dexamethasone + cisplatin + etoposide + ifosfamide) and 45 patients treated with radiotherapy alone found that the CR rate was higher using chemoradiotherapy than radiotherapy alone $(90.9 \%$ vs. $77.8 \%$, respectively; $\mathrm{p}=0.124)$, as was the 5 -year OS rate $(82 \%$ vs. $49 \%$, respectively; $p<0.001$ ) [26]. In our current study, we specifically investigated the sequence and appropriate timing of the radiotherapy and chemotherapy combinations.

Kwong et al. [40] conducted an international multicenter retrospective cohort study comparing sequential chemoradiotherapy to CCRT in patients with early-stage ENKTL. In their study, 54 patients received sequential chemoradiotherapy (chemotherapy + radiotherapy) and 190 received CCRT (CCRT \pm chemotherapy); none received anthracycline-containing regimens. No significant differences in the CR, PFS, and OS rates were observed between the sequential chemoradiotherapy and CCRT groups. Additionally, Moon et al. [41] enrolled 158 stage IE-IIE patients treated with non-anthracycline-based regimens, of whom 61 received sequential chemoradiotherapy (chemotherapy + radiotherapy) and 55 received CCRT (CCRT + chemotherapy). The CR and 5-year OS rates did not differ between the sequential chemoradiotherapy and CCRT groups $(82.0 \%$ vs. $74.5 \%$, respectively, $p=0.334$; and $77.7 \pm 5.5 \%$ vs. 68.9 $\pm 6.8 \%$, respectively, $p=0.234$ ). These findings indicate that both sequential chemoradiotherapy and CCRT are effective in treating early-stage ENKTL, and produce similar outcomes. Consistent with these studies, our own investigation showed that scheduling chemotherapy and radiotherapy in sequential vs. sandwich routines did not affect the survival outcomes of patients with localized ENKTL. In contrast, another retrospective cohort study conducted by Cao et al. [42] showed that CCRT was associated with a better survival outcome than sequential chemoradiotherapy in limited-stage patients. These conflicting results may be attributable to the fact that patients received less effective $\mathrm{CHOP}$ or CHOP-like regimens in Cao et al.'s study. CCRT increases tumor sensitivity to the CHOP regimen, rendering it more effective than sequential chemoradiotherapy. The regimens used in our study have been shown to be more effective than CHOP or CHOP-like regimens for treating ENKTL. Consequently, the radiotherapy scheduling sequence (before, after, or in between chemotherapy) did not significantly impact the outcome.

Treatment-related adverse events were generally consistent in patients undergoing the sequential and sandwich groups, with the exception that grade $3 / 4$ hematological toxicities were slightly more common in the sandwich group. Both sequential and sandwich chemoradiotherapy regimens were tolerated for the treatment of early-stage ENKTL.

Several studies [43-45] have identified local invasion as the most significant factor related to poor survival. Kim et al. [43] reported that the presence of local tumor invasion predicted an inferior $\mathrm{CR}$ rate and poor survival outcomes in patients with stage IE-IIE ENKTL of the upper aerodigestive tract. Consistent with these findings, our study found that local invasion (i.e., tumors invading adjacent bone, skin, and soft tissues) was the most significant predictor of poorer OS and PFS rates. The PINK and PINK-E scoring systems were proposed by Kim et al. [35] in 2016 as a prognostic index for ENKTL patients receiving non-anthracycline-containing regimens. The PINK model comprises 4 independent risk factors: $>60$ years of age, advanced-stage disease, distant lymph node invasion, and non-nasal type disease. The PINK-E (comprising the 4 independent risk factors of PINK combined with pretreatment plasma EBV-DNA copy number) also predicts survival. In their study, the 3-year OS rates in the low-, intermediate-, and high-risk groups were $81 \%, 62 \%$, and $25 \%$ using the PINK model, and $81 \%, 55 \%$, and $28 \%$ using the PINK-E model, respectively. Our results showed that the 3-year OS rates of both the low- and intermediate-risk groups were consistent with those of Kim et al. [35]; however, PINK and PINK-E had no predictive significance for survival outcomes on multivariate analysis. This may be due to the limited number of elderly patients (aged $>60$ years) $(N=18$; $8.4 \%)$, a lack of distant lymph node invasion $(N=2$; $0.9 \%)$, and the fact that baseline EBV-DNA data were unavailable for $>50 \%$ of the patients $(N=121)$.

Our study was a multi-center investigation; however, there were certain limitations in this study. It was a retrospective analysis comprising a relatively small sample size; moreover, patients received non-uniform chemotherapy regimens and were administered varying radiotherapy dosages.

In conclusion, our results showed that, in the era of L-asparaginase/pegaspargase, the timing and sequence of chemotherapy and radiotherapy may not affect the therapeutic responses or survival outcomes in newly diagnosed patients with stage IE-IIE ENKTL as long as both are used in combination. Future large-scale, prospective, multicenter studies are required to validate these findings.

\section{Abbreviations}

ENKTL: extranodal natural killer/T-cell lymphoma, nasal type; CR: complete response; MDR: multi-drug resistance; CHOP: cyclophosphamide, anthracycline, vincristine, and prednisone; CCRT: 
concurrent chemoradiotherapy; CT: computed tomography; MRI: magnetic resonance imaging; PET: positron emission tomography; ECOG: Eastern Cooperative Oncology Group; LDH: lactate dehydrogenase; EBV: Epstein-Barr virus; PINK: prognostic index for natural killer/T-cell lymphoma; GELOX: gemcitabine, oxaliplatin, and L-asparaginase/ pegaspargase; SMILE: dexamethasone, methotrexate, ifosfamide, L-asparaginase/pegaspargase, and etoposide; VLP: vincristine, L-asparaginase/ pegaspargase, and prednisone; OS: overall survival; PFS: progression-free survival; PR: partial response; CT+RT: chemotherapy followed by radiotherapy; $\mathrm{RT}+\mathrm{CT}$ : radiotherapy followed by chemotherapy; PD: progressive disease; SD: stable disease; HR: hazard ratio; CI: confidence interval.

\section{Supplementary Material}

Supplementary figures and tables. http://www.jcancer.org/v09p1598s1.pdf

\section{Acknowledgements}

This study was supported by grants from the Hunan Provincial Science and Technology Department (No. 2016JJ3083) and the Heath and Family Planning Commission of Hunan Province (No. c2015-52).

\section{Competing Interests}

The authors have declared that no competing interest exists.

\section{References}

1. Vose J, Armitage J, Weisenburger D. International peripheral T-cell and natural killer/T-cell lymphoma study: pathology findings and clinical outcomes. Journal of clinical oncology: official journal of the American Society of Clinical Oncology. 2008; 26: 4124-30.

2. Au WY, Weisenburger DD, Intragumtornchai T, Nakamura S, Kim WS, Sng I, et al. Clinical differences between nasal and extranasal natural killer/T-cell lymphoma: a study of 136 cases from the International Peripheral T-Cell Lymphoma Project. Blood. 2009; 113: 3931-7.

3. Lee J, Park YH, Kim WS, Lee SS, Ryoo BY, Yang SH, et al. Extranodal nasal type NK/T-cell lymphoma: elucidating clinical prognostic factors for risk-based stratification of therapy. European journal of cancer (Oxford, England: 1990). 2005; 41: 1402-8.

4. Cheung MM, Chan JK, Lau WH, Ngan RK, Foo WW. Early stage nasal NK/T-cell lymphoma: clinical outcome, prognostic factors, and the effect of treatment modality. International journal of radiation oncology, biology, physics. 2002; 54: 182-90.

5. Ma HH, Qian LT, Pan HF, Yang L, Zhang HY, Wang ZH, et al. Treatment outcome of radiotherapy alone versus radiochemotherapy in early stage nasal natural killer/T-cell lymphoma. Medical oncology (Northwood, London, England). 2010; 27: 798-806

6. Wu RY, Liu K, Wang WH, Jin J, Song YW, Wang SL, et al. Patterns of Primary Tumor Invasion and Regional Lymph Node Spread Based on Magnetic Resonance Imaging in Early-Stage Nasal NK/T-cell Lymphoma: Implications for Clinical Target Volume Definition and Prognostic Significance. International journal of radiation oncology, biology, physics. 2017; 97: 50-9.

7. Kim SJ, Kim WS. Treatment of localized extranodal NK/T cell lymphoma, nasal type. International journal of hematology. 2010; 92: 690-6.

8. Li YX, Yao B, Jin J, Wang WH, Liu YP, Song YW, et al. Radiotherapy as primary treatment for stage IE and IIE nasal natural killer/T-cell lymphoma. Journal of clinical oncology: official journal of the American Society of Clinical Oncology. 2006; 24: 181-9.

9. Wang ZY, Li YX, Wang WH, Jin J, Wang H, Song YW, et al. Primary radiotherapy showed favorable outcome in treating extranodal nasal-type NK/T-cell lymphoma in children and adolescents. Blood. 2009; 114: 4771-6.
10. Kim WS, Song SY, Ahn YC, Ko YH, Baek CH, Kim DY, et al. CHOP followed by involved field radiation: is it optimal for localized nasal natural killer/T-cell lymphoma? Annals of oncology: official journal of the European Society for Medical Oncology. 2001; 12: 349-52.

11. Wang B, Lu JJ, Ma X, Guo Y, Lu H, Hong X, et al. Combined chemotherapy and external beam radiation for stage IE and IIE natural killer T-cell lymphoma of nasal cavity. Leukemia \& lymphoma. 2007; 48: 396-402.

12. Kwong YL. Natural killer-cell malignancies: diagnosis and treatment. Leukemia. 2005; 19: 2186-94.

13. Kim BS, Kim TY, Kim CW, Kim JY, Heo DS, Bang YJ, et al. Therapeutic outcome of extranodal NK/T-cell lymphoma initially treated with chemotherapy--result of chemotherapy in NK/T-cell lymphoma. Acta oncologica (Stockholm, Sweden). 2003; 42: 779-83.

14. Drenou B, Lamy T, Amiot L, Fardel O, Caulet-Maugendre S, Sasportes M, et al. CD3- CD56+ non-Hodgkin's lymphomas with an aggressive behavior related to multidrug resistance. Blood. 1997; 89: 2966-74.

15. Yong $W$. Clinical study of l-asparaginase in the treatment of extranodal NK/T-cell lymphoma, nasal type. Hematological oncology. 2016; 34: 61-8.

16. Pokrovsky VS, Vinnikov D. L-Asparaginase for newly diagnosed extra-nodal NK/T-cell lymphoma: systematic review and meta-analysis. Expert review of anticancer therapy. 2017; 17: 759-68.

17. Ando M, Sugimoto K, Kitoh T, Sasaki M, Mukai K, Ando J, et al. Selective apoptosis of natural killer-cell tumours by l-asparaginase. British journal of haematology. 2005; 130: 860-8.

18. Yamaguchi M, Kwong YL, Kim WS, Maeda Y, Hashimoto C, Suh C, et al. Phase II study of SMILE chemotherapy for newly diagnosed stage IV, relapsed, or refractory extranodal natural killer (NK)/T-cell lymphoma, nasal type: the NK-Cell Tumor Study Group study. Journal of clinical oncology: official journal of the American Society of Clinical Oncology. 2011; 29: 4410-6.

19. Kwong YL, Kim WS, Lim ST, Kim SJ, Tang T, Tse E, et al. SMILE for natural killer/T-cell lymphoma: analysis of safety and efficacy from the Asia Lymphoma Study Group. Blood. 2012; 120: 2973-80.

20. Jaccard A, Gachard N, Marin B, Rogez S, Audrain M, Suarez F, et al. Efficacy of L-asparaginase with methotrexate and dexamethasone (AspaMetDex regimen) in patients with refractory or relapsing extranodal NK/T-cell lymphoma, a phase 2 study. Blood. 2011; 117: 1834-9.

21. NCCN guidelines Version 2. 2018 T-Cell Lymphomas.

22. Yamaguchi $M$, Tobinai $K$, Oguchi $M$, Ishizuka $N$, Kobayashi $Y$, Isobe $Y$, et al. Concurrent chemoradiotherapy for localized nasal natural killer/T-cell lymphoma: an updated analysis of the Japan clinical oncology group study JCOG0211. Journal of clinical oncology: official journal of the American Society of Clinical Oncology. 2012; 30: 4044-6.

23. Kim SJ, Kim K, Kim BS, Kim CY, Suh C, Huh J, et al. Phase II trial of concurrent radiation and weekly cisplatin followed by VIPD chemotherapy in newly diagnosed, stage IE to IIE, nasal, extranodal NK/T-Cell Lymphoma: Consortium for Improving Survival of Lymphoma study. Journal of clinical oncology: official journal of the American Society of Clinical Oncology. 2009; 27: 6027-32.

24. Kim SJ, Yang DH, Kim JS, Kwak JY, Eom HS, Hong DS, et al. Concurrent chemoradiotherapy followed by L-asparaginase-containing chemotherapy, VIDL, for localized nasal extranodal NK/T cell lymphoma: CISL08-01 phase II study. Annals of hematology. 2014; 93: 1895-901.

25. Yamaguchi M, Suzuki R, Oguchi M, Asano N, Amaki J, Akiba T, et al. Treatments and Outcomes of Patients With Extranodal Natural Killer/T-Cell Lymphoma Diagnosed Between 2000 and 2013: A Cooperative Study in Japan. Journal of clinical oncology: official journal of the American Society of Clinical Oncology. 2017; 35: 32-9.

26. Dong LH, Zhang LJ, Wang WJ, Lei W, Sun X, Du JW, et al. Sequential DICE combined with l-asparaginase chemotherapy followed by involved field radiation in newly diagnosed, stage IE to IIE, nasal and extranodal NK/T-cell lymphoma. Leukemia \& lymphoma. 2016; 57: 1600-6.

27. Aviles A, Neri N, Fernandez R, Huerta-Guzman J, Nambo MJ. Combined therapy in untreated patients improves outcome in nasal NK/T lymphoma: results of a clinical trial. Medical oncology (Northwood, London, England). 2013; 30: 637.

28. Jiang M, Zhang H, Jiang Y, Yang Q, Xie L, Liu W, et al. Phase 2 trial of "sandwich" L-asparaginase, vincristine, and prednisone chemotherapy with radiotherapy in newly diagnosed, stage IE to IIE, nasal type, extranodal natural killer/T-cell lymphoma. Cancer. 2012; 118: 3294-301.

29. Zhang L, Jiang M, Xie L, Zhang H, Jiang Y, Yang QP, et al. Five-year analysis from phase 2 trial of "sandwich" chemoradiotherapy in newly diagnosed, stage IE to IIE, nasal type, extranodal natural killer/T-cell lymphoma. Cancer medicine. 2016; 5: 33-40.

30. Wang L, Wang ZH, Chen XQ, Li YJ, Wang KF, Xia YF, et al. First-line combination of gemcitabine, oxaliplatin, and L-asparaginase (GELOX) followed by involved-field radiation therapy for patients with stage IE/IIE extranodal natural killer/T-cell lymphoma. Cancer. 2013; 119: 348-55.

31. Jaffe ES. The 2008 WHO classification of lymphomas: implications for clinical practice and translational research. Hematology American Society of Hematology Education Program. 2009: 523-31.

32. Jiang M, Bennani NN, Feldman AL. Lymphoma classification update: T-cell lymphomas, Hodgkin lymphomas, and histiocytic/dendritic cell neoplasms. Expert review of hematology. 2017; 10: 239-49.

33. Rosenberg SA. Validity of the Ann Arbor staging classification for the non-Hodgkin's lymphomas. Cancer treatment reports. 1977; 61: 1023-7. 
34. Cheson BD, Fisher RI, Barrington SF, Cavalli F, Schwartz LH, Zucca E, et al. Recommendations for initial evaluation, staging, and response assessment of Hodgkin and non-Hodgkin lymphoma: the Lugano classification. Journal of clinical oncology: official journal of the American Society of Clinical Oncology. 2014; 32: 3059-68.

35. Kim SJ, Yoon DH, Jaccard A, Chng WJ, Lim ST, Hong H, et al. A prognostic index for natural killer cell lymphoma after non-anthracycline-based treatment: a multicentre, retrospective analysis. The Lancet Oncology. 2016; 17: 389-400.

36. Trotti A, Colevas AD, Setser A, Rusch V, Jaques D, Budach V, et al. CTCAE v3.0: development of a comprehensive grading system for the adverse effects of cancer treatment. Seminars in radiation oncology. 2003; 13: 176-81.

37. Huang L, Yuan B, Wu H, Chu H, Liu Y, Wu S, et al. Comparative Study of L-Asparaginase-Based LOP Regimen Over CHOP Regimen Before Radiotherapy for Stage IIE Extranodal Nasal Type NK/T Cell Lymphoma: A Study of 2 Centers. Clinical lymphoma, myeloma \& leukemia. 2017; 17: 152-8.

38. Yang L, Liu H, Xu XH, Wang XF, Huang HM, Shi WY, et al. Retrospective study of modified SMILE chemotherapy for advanced-stage, relapsed, or refractory extranodal natural killer (NK)/T cell lymphoma, nasal type. Medical oncology (Northwood, London, England). 2013; 30: 720.

39. Yong W, Zheng W, Zhu J, Zhang Y, Wei Y, Wang X, et al. Midline NK/T-cell lymphoma nasal-type: treatment outcome, the effect of L-asparaginase based regimen, and prognostic factors. Hematological oncology. 2006; 24: 28-32.

40. Kwong YL, Kim SJ, Tse E, Oh SY, Kwak JY, Eom HS, et al. Sequential chemotherapy/radiotherapy was comparable with concurrent chemoradiotherapy for stage I/II NK/T-cell lymphoma. Annals of oncology: official journal of the European Society for Medical Oncology. 2017.

41. Moon JH, Lee BH, Kim JA, Lee YJ, Chae YS, Yhim HY, et al. Clinical impact of induction treatment modalities and optimal timing of radiotherapy for the treatment of limited-stage NK/T cell lymphoma. Leukemia research. 2016; 49: 80-7.

42. Cao J, Lan S, Shen L, Si H, Zhang N, Li H, et al. A comparison of treatment modalities for nasal extranodal natural killer/T-cell lymphoma in early stages: The efficacy of CHOP regimen based concurrent chemoradiotherapy. Oncotarget. 2017; 8: 20362-70.

43. Kim TM, Park YH, Lee SY, Kim JH, Kim DW, Im SA, et al. Local tumor invasiveness is more predictive of survival than International Prognostic Index in stage I(E)/II(E) extranodal NK/T-cell lymphoma, nasal type. Blood. 2005; 106: 3785-90.

44. Kim JY, Lee SW, Lee JH, Suh C, Yoon DH, Lee BJ, et al. Stage IE/IIE extranodal NK/T-cell lymphoma arising in the nasal cavity: analysis of CT findings and their prognostic value. Clinical radiology. 2013; 68: e384-90.

45. Yang CW, Wang CW, Hong RL, Tsai CL, Yao M, Tang JL, et al. Treatment outcomes of and prognostic factors for definitive radiotherapy with and without chemotherapy for Stage I/II nasal extranodal NK/T-cell lymphoma. Journal of radiation research. 2017; 58: 114-22. 